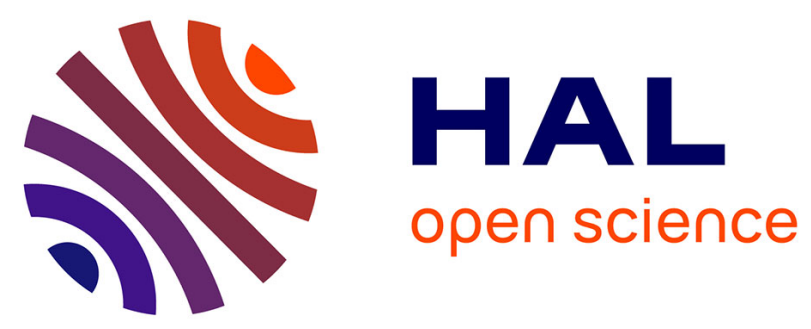

\title{
Les institutions européennes confrontées à la définition des limites de l'Europe pour leurs propres frontières
}

Thibault Courcelle

\section{To cite this version:}

Thibault Courcelle. Les institutions européennes confrontées à la définition des limites de l'Europe pour leurs propres frontières. Bertrand Vayssière. Penser les frontières européennes au XXIe siècle: réflexion croisée des sciences sociales, Peter Lang, pp.55-72, 2015, Europe des cultures, 978-2-87574266-7. hal-01517089

\section{HAL Id: hal-01517089 \\ https://hal-univ-tlse2.archives-ouvertes.fr/hal-01517089}

Submitted on 2 May 2017

HAL is a multi-disciplinary open access archive for the deposit and dissemination of scientific research documents, whether they are published or not. The documents may come from teaching and research institutions in France or abroad, or from public or private research centers.
L'archive ouverte pluridisciplinaire HAL, est destinée au dépôt et à la diffusion de documents scientifiques de niveau recherche, publiés ou non, émanant des établissements d'enseignement et de recherche français ou étrangers, des laboratoires publics ou privés. 


\section{Les institutions européennes confrontées à la définition des limites de l'Europe pour leurs propres frontières.}

Thibault COURCELLE, Maître de conférences en géographie, Centre universitaire J.-F. Champollion d'Albi, LISST CNRS (UMR 5193), Centre interdisciplinaire d'études urbaines (CIEU).

Dans la réflexion sur les frontières européennes, relativement peu de travaux s'intéressent aux liens qui unissent les institutions européennes à la définition des frontières de l'Europe. Nous prendrons ici en compte les deux institutions qui se revendiquent comme étant spécifiquement européennes : l'Union européenne (UE) et le Conseil de l'Europe. L'UE compte 28 membres depuis l'adhésion de la Croatie en 2013 et le Conseil de l'Europe, avec 47 membres, est considéré comme étant l'organisation de la «grande Europe» de l'Atlantique au Pacifique. Nous laissons volontairement de côté l'Organisation pour la Sécurité et la Coopération en Europe (OSCE) qui, bien qu'ayant le nom d'Europe dans sa dénomination, n'est pas spécifiquement européenne puisqu'elle comprend également comme membres des Etats d'Amérique (Etats-Unis et Canada) et d'Asie (Mongolie, ainsi que tous les Etats d'Asie Centrale ex-soviétiques). A l'inverse, l'UE et le Conseil de l'Europe n'ont comme membres que des Etats précisément européens et c'est d'ailleurs inscrit dans leurs statuts.

L'article 49 du traité sur l'UE définit ainsi les conditions pour devenir membre de l'organisation : "Tout État européen qui respecte les valeurs visées à l'article 2 et s'engage à les promouvoir peut demander à devenir membre de l'Union. »"

Les valeurs définies dans l'article 2 sont les suivantes : «L'Union est fondée sur les valeurs de respect de la dignité humaine, de liberté, de démocratie, d'égalité, de l'État de droit, ainsi que de respect des droits de l'homme, y compris des droits des personnes appartenant à des minorités. Ces valeurs sont communes aux États membres dans une société caractérisée par le pluralisme, la nondiscrimination, la tolérance, la justice, la solidarité et l'égalité entre les femmes et les hommes. »

Deux critères sont ainsi posés : l'un géographique, le second politique ou idéologique. Cependant, si cet article 2 et plusieurs autres énumèrent ce que sont les valeurs communes européennes, aucune définition géographique de ce que l'on entend par «Etat européen » ou «Europe » n'est présente. Ce flou géographique est perceptible dans les traités constitutifs de la CEE et de l'UE depuis l'adoption du traité de Rome en 1957. Il n'est pas clarifié lors de l'adoption des critères de Copenhague en 1993 qui définissent un ensemble de conditions politiques (présence d'institutions stables garantissant la démocratie, l'État de droit, les droits de l'homme, le respect des minorités et leur protection), économiques (l'existence d'une économie de marché viable et la capacité à faire face aux forces du marché et à la pression concurrentielle à l'intérieur de l'Union) et juridiques avec l'adoption de l'acquis communautaire. Le critère géographique est totalement absent.

Le même flou est également remarquable dans les statuts de la plus ancienne organisation politique européenne : le Conseil de l'Europe créé en 1949. Les articles 3 et 4, qui définissent les conditions pour en devenir membre, posent les deux mêmes critères, géographique et politique/idéologique. Le second critère est bien détaillé dans l'article $3:$ «Tout membre du Conseil de l'Europe reconnaît le principe de la prééminence du droit et le principe en vertu duquel toute personne placée sous sa juridiction doit jouir des droits de l'homme et des libertés fondamentales. Il $s^{\prime}$ engage à collaborer sincèrement et activement à la poursuite du but défini au chapitre Ier. $»^{2} \mathrm{Si}$

\footnotetext{
${ }^{1}$ Site du journal officiel de l'UE : http://eur-lex.europa.eu/legal-content/FR/TXT/?uri=CELEX:12012M/TXT

${ }^{2}$ Statuts du Conseil de l'Europe sur le site du bureau des traités :

http://conventions.coe.int/Treaty/fr/treaties/html/001.htm
} 
les valeurs prônées par le Conseil de l'Europe sont peu ou prou les mêmes que celles de l'UE, le critère géographique n'est lui pas du tout détaillé dans l'article 4 : "Tout Etat européen considéré comme capable de se conformer aux dispositions de l'article 3 et comme en ayant la volonté peut être invité par le Comité des Ministres à devenir membre du Conseil de l'Europe. Tout Etat ainsi invité aura la qualité de membre dès qu'un instrument d'adhésion au présent Statut aura été remis en son nom au Secrétaire Général. »

Alors pourquoi cette absence de définition géographique et culturelle de ce qu'est un Etat européen dans les statuts des institutions européennes ? En quoi cette absence a-t-elle pu poser problème lors des élargissements à l'Est du Conseil de l'Europe et de l'UE et comment ces institutions y ont remédié ?

Il convient de rappeler succinctement ici que les institutions européennes sont pleinement confrontées à la difficulté des géographes et des historiens de définir les limites de l'Europe en particulier à l'Est et au Sud-Est car l'Europe n'est pas un continent. Elle ne forme qu'un seul ensemble continental avec l'Asie que l'on nomme l'Eurasie. Le sociologue Edgar Morin avait ainsi rappelé, dans son ouvrage «Penser l'Europe » en 1987, la difficulté à définir ce qu'est l'Europe à partir des frontières géographiques ou historiques : "L'Europe est une notion géographique sans frontières avec l'Asie et une notion historique aux frontières changeantes. C'est une notion aux multiples visages que l'on ne saurait surimpressionner les uns les autres sans créer le flou ${ }^{3}$.

Si l'Europe de l'Ouest est géographiquement et culturellement clairement définie par les coupures que représentent l'Océan Atlantique et la Mer Méditerranée, la délimitation de l'Europe orientale est un véritable «casse-tête » pour les géographes et pour les institutions européennes.

\section{I - Une problématique de fin de guerre froide pour les institutions européennes.}

Durant plusieurs décennies, la problématique de la délimitation de l'Europe ne s'est pas véritablement posée pour les institutions européennes puisque celles-ci étaient formellement cantonnées à l'Ouest du rideau de fer, donc dans la partie la plus distinctement circonscrite de l'Europe par la Mer du Nord, l'Océan Atlantique et la Mer Méditerranée. La question des limites de l'Europe et des critères d'européanité semblait «aller alors de soi » lors des élargissements, et l'Europe « de l'Atlantique à l'Oural » que prônait de manière assez provocatrice le général de Gaulle en pleine guerre froide en $1959^{4}$ était bien éloignée de la réalité géopolitique de l'époque.

Pourtant, dès les premiers élargissements du Conseil de l'Europe, la Turquie devient membre de l'organisation en 1950 quelques mois seulement après sa création en $1949^{5}$. Ce pays, dont seulement 3\% du territoire, la Thrace, est situé géographiquement en Europe, n'a alors, dans le contexte géopolitique tendu de début de guerre froide, suscité aucune controverse lors de son adhésion quant au caractère européen ou non de cet Etat. La candidature actuelle de la Turquie à l'UE et l'ouverture des négociations d'adhésion depuis 2005 suscite pourtant de nombreuses polémiques à propos de son appartenance géographique et culturelle à l'Europe. En 1950, les préoccupations concernant les menaces potentielles liées à l'islamisme étaient alors inexistantes, et les Etats-Unis, l'un des principaux soutiens politiques et financiers du Conseil de l'Europe lors de sa création, ont toujours soutenu l'arrimage de la Turquie dans le camp occidental et son adhésion aux institutions européennes. Et, bien que l'Etat turc ait violé plusieurs fois les valeurs communes du

\footnotetext{
${ }^{3}$ MORIN E., Penser l'Europe, Gallimard, Paris, 1987, p. 27.

${ }^{4}$ La formule est tirée du discours de Charles de Gaulle prononcé à Strasbourg en novembre 1959 : « Oui, c'est l'Europe, depuis l'Atlantique jusqu'à l'Oural, c'est l'Europe, c'est toute l'Europe, qui décidera du destin du monde ! », in : http://www.gaullisme.fr/2010/06/15/leurope-de-latlantique-a-loural/

${ }^{5}$ Une erreur volontaire de date indique 1949 dans les documents officiels, pour que la date d'adhésion de la Turquie corresponde symboliquement à celle de la Grèce.
} 
Conseil de l'Europe, notamment lors des coups d'Etat militaires de 1960 et de 1980, il n'a, par son importance stratégique durant la guerre froide et sa place au sein de l'OTAN, jamais été exclu et a toujours été considéré comme étant un Etat pleinement européen par cette organisation.

Le cas de l'île de Chypre, devenue membre du Conseil de l'Europe en 1961, peu de temps après son indépendance de la couronne britannique, est également intéressant. La question de son appartenance à l'Europe, alors que l'île est géographiquement considérée comme étant asiatique dans tous les atlas, n'a pourtant posé aucun problème. La population chypriote étant majoritairement culturellement grecque a fait que l'adhésion de la République de Chypre n'a suscité aucun débat sur son "européanité » à cette époque. Il n'y a pas eu non plus de débats sur l'appartenance de Chypre à l'Europe lors de son adhésion à l'UE en 2004. Tous les débats se sont alors focalisés sur les tentatives de réunification de l'île, sous l'égide de l'ONU et du plan Annan, proposant la création d'une Confédération chypriote, plan massivement rejeté par les chypriotes grecs de la partie sud de l'île.

Les élargissements du Conseil de l'Europe et de la CEE durant la guerre froide n'ont pas soulevé de problèmes spécifiquement géographiques et culturels aux institutions européennes. A la veille de la chute du Mur de Berlin en 1989, le Conseil de l'Europe comprend vingt-quatre membres, soit la totalité des Etats de l'Europe occidentale, et la CEE en compte douze.

\section{Les institutions européennes à la veille de la chute du mur}

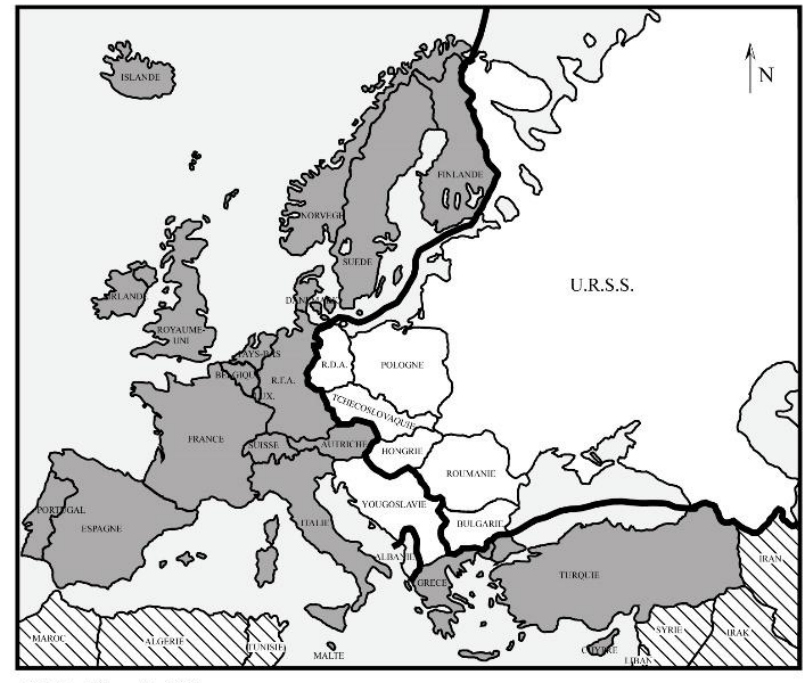

Thibault Courcelle, 2014

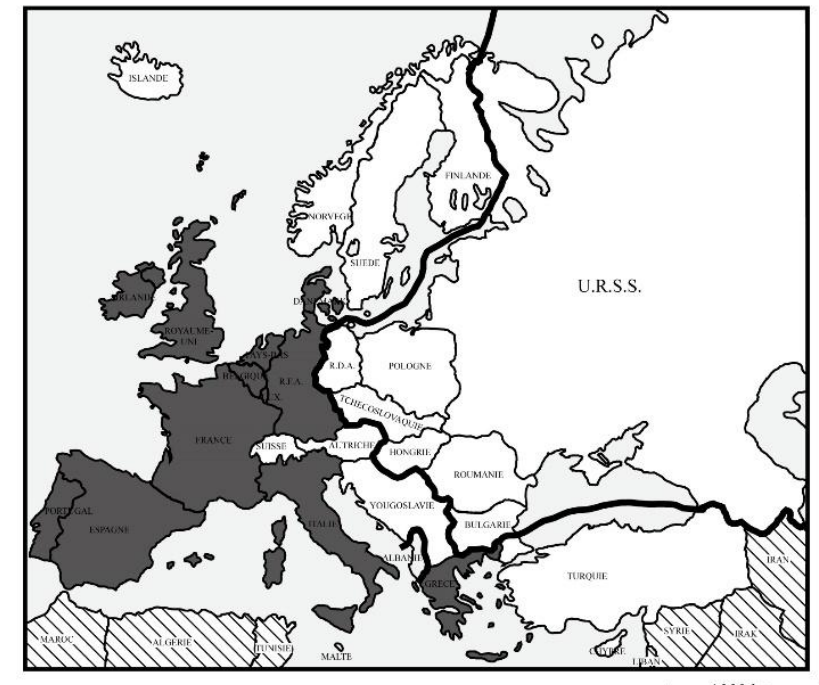

$1000 \mathrm{~km}$

Pays membres du Conseil de l'Europe

Pays membres de la Communauté économique européenne (CEE)

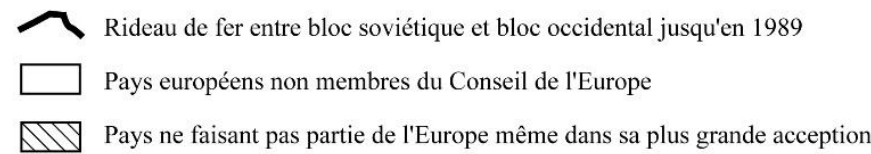

La fin de la guerre froide bouleverse complètement la donne européenne avec notamment l'effondrement des régimes communistes à l'Est et la création de quinze nouveaux Etats issus des républiques soviétiques. Le Conseil de l'Europe, en invitant Mikhaïl Gorbatchev à prononcer un discours historique à l'Assemblée parlementaire le 5 juillet 1989 où celui-ci développera son fameux concept de la «maison commune européenne », prend part aux évènements aboutissants à la chute du mur.

Tous les Etats d'Europe centrale et orientale se tournent alors vers les institutions européennes et manifestent leur souhait d'une intégration rapide et d'une adhésion aux valeurs 
communes de l'Ouest. La CEE étant en pleine phase de renforcement de son intégration avec la création de l'UE par la préparation du traité de Maastricht, les chefs d'Etats et de gouvernements tranchent pour l'approfondissement plutôt que l'élargissement rapide à l'Est. Le Conseil de l'Europe devient alors de facto «l'antichambre de l'UE» pour tous ces Etats ${ }^{6}$. Et, suite à l'adhésion de la Hongrie dès 1990 et de la totalité des Etats d'Europe centrale en trois ans de 1990 à 1992, le Conseil de l'Europe se trouve confronté à un grand nombre de demandes d'adhésion provenant de l'Europe orientale. Dès 1992, la Fédération de Russie demande son adhésion, suivie des ex-républiques soviétiques y compris d'Asie Centrale. La question de la définition de critères géographiques et culturels se pose alors de manière pressante au sein du Conseil de l'Europe. Cette question de la délimitation de l'Europe à l'Est est alors récurrente dans tous les débats de l'Assemblée parlementaire sur l'élargissement entre 1992 et 1994. Ces débats se basent notamment sur les rapports de la Commission des questions politiques de l'Assemblée, présentés par son président, le député chrétien-démocrate allemand Gerhard Reddemann. Celui-ci résume, en 1994, les quatre principales représentations qui opposent les parlementaires concernant les limites de l'Europe à l'Est :

"Il y a d'abord celle qui exclut catégoriquement la Russie et qui estime que cette dernière ne devrait pas pouvoir devenir membre du Conseil de l'Europe, au motif que l'appartenance à l'Organisation d'une seule des deux grandes puissances de la seconde moitié du siècle risquerait d'en perturber l'équilibre politique. D'après la deuxième école, qui plaide pour des frontières plus restreintes, l'Europe ne devrait pas inclure le Caucase. La troisième école, qui considère que le Caucase a toujours fait partie de l'Europe, comprend deux courants, dont le premier propose d'inclure les trois Etats caucasiens alors que l'autre n'en retient que deux [Géorgie et Arménie]. Enfin, pour la quatrième école, les anciennes républiques islamiques de l'ex-Union Soviétique feraient également partie de l'Europe. ${ }^{7}$

Il ne faudra pas moins de trois rapports $^{8}$, tous présentés par M. Reddemann, ainsi que les avis $^{9}$ de la Commission des questions juridiques et des droits de l'homme et de la Commission des relations avec les pays européens non membres, pour que l'Assemblée parlementaire adopte une recommandation, la Recommandation 1247, fixant les critères d'européanité des Etats et les limites aux élargissement du Conseil de l'Europe. Cette recommandation confirme le caractère européen du Conseil de l'Europe : "Ne peuvent en principe devenir membres du Conseil de l'Europe que des Etats dont le territoire national est situé en totalité ou en partie sur le continent européen et dont la culture est étroitement liée à la culture européenne. " ${ }^{10}$

L'Assemblée énumère ensuite une liste de tous les Etats qui rentrent dans cette définition du concept «européen». Il ressort des débats entre parlementaires que le critère culturel ou «communauté de valeurs culturelles » est très contesté et peu pertinent pour définir l'«européanité » d'un Etat, mais que c'est plutôt l'existence d'un "sentiment d'appartenance à l'Europe » ou « la volonté affirmée d'en faire partie » qui prime :

"A défaut d'un contenu très clair pour les soi-disant valeurs communes, il existe, selon certains parlementaires, un facteur qui peut constituer un critère convaincant : c'est l'existence d'un sentiment d'appartenance à l'Europe ou la volonté affirmée de faire partie de l'Europe. Cet élément n'est pas sans rappeler la pensée d'Ernest Renan qui définissait la nation comme " un

\footnotetext{
${ }^{6}$ COURCELLE T., « Chapitre II : Le Conseil de l'Europe, une « antichambre » de l'Union européenne », in : Le Conseil de l'Europe : enjeux et représentations, Thèse de doctorat, Institut Français de Géopolitique, Université Paris 8, 2008, p. 73 à 138.

${ }^{7}$ Gerhard Reddemann à la tribune de l'Assemblée parlementaire le 26 janvier 1994, In : HUBER D., Une décennie pour l'Histoire : le Conseil de l'Europe 1989 - 1999, Editions du Conseil de l'Europe, Strasbourg - p.115.

${ }^{8}$ Documents 6629 (1992), 6975 (1993) et 7103 (1994) de l'Assemblée parlementaire du Conseil de l'Europe.

${ }^{9}$ Documents 7148 et 7166 (1994) de l'Assemblée parlementaire du Conseil de l'Europe.

${ }^{10}$ Recommandation 1247 (1994) de l'Assemblée parlementaire du Conseil de l'Europe.
} 
plébiscite de tous les jours » ou celle d'Altiero Spinelli qui souligne l'importance de l'adhésion des peuples à l'idée européenne comme composante de l'identité européenne. " ${ }^{11}$

C'est donc sur cette base assez vague de «sentiment d'appartenance à l'Europe » ou de « volonté affirmée » d'en faire partie, que l'Assemblée décide finalement de donner aux trois Etats du Caucase la possibilité d'adhérer : "En raison de leurs liens culturels avec l'Europe, l'Arménie, l'Azerbaïdjan et la Géorgie auraient la possibilité de demander leur adhésion à condition qu'ils indiquent clairement leur volonté d'être considérés comme faisant partie de l'Europe. ${ }^{12}$

L'emploi du conditionnel dans cette décision, très rare dans un texte européen, est révélateur du difficile compromis finalement trouvé à l'Assemblée où, durant deux ans, les parlementaires se sont confrontés à l'aide d'atlas qui, selon les maisons d'édition, incluaient ou excluaient les Etats du Caucase à l'Europe. Ces trois Etats ont été traités ensemble en raison du lobbying permanent de la Turquie, déjà membre de l'organisation et proche de l'Azerbaïdjan qui appartient à la même famille linguistique, alors que de nombreux parlementaires étaient plus favorable au cas de l'Arménie, en raison des liens d'affinités culturelles liés à l'histoire et à l'importante diaspora arménienne dans les pays d'Europe occidentale. Dans toutes les discussions sur les critères d'européanité, la Turquie a toujours veillé à ce que l'Arménie ne soit pas traitée plus avantageusement que l'Azerbaïdjan ${ }^{13}$.

La dernière catégorie, celle des Etats d'Asie centrale issus de l'ex-Union soviétique, ne rentre donc finalement pas dans la définition d'Etats « européens », bien que l'un d'entre eux, le Kazakhstan, ait une partie de son territoire en Europe d'après la limite fixée en 1703 de l'Oural à la Caspienne par le tsar russe Pierre Le Grand et son cartographe Vassili Tatichtchev ${ }^{14}$. Certains parlementaires font d'ailleurs remarquer que le Kazakhstan, peuplé d'une importante minorité de Russes (près d'un quart de la population totale), pourrait être considéré comme « européen » et d'autres que l'OSCE inclut toutes les ex-républiques de l'URSS sans distinction. La recommandation fixant les limites géographiques et culturelles de l'Europe est finalement adoptée à l'unanimité par l'Assemblée en 1994, ce qui lui confère une forte légitimité.

Finalement, en s'appuyant sur cette recommandation, l'Assemblée parlementaire et le Comité des Ministres vont progressivement intégrer l'ensemble des Etats d'Europe orientale au Conseil de l'Europe en moins de dix ans, dont la Fédération de Russie dès 1996, et les Etats du Caucase en 1999 (Géorgie) et 2001 (Arménie et Azerbaïdjan), qui ont fait le choix de se considérer comme étant des pays européens plutôt qu'asiatiques.

\footnotetext{
${ }^{11}$ BITSCH M.-T., « L'élargissement du Conseil de l'Europe vers l'est : les débats sur l'appartenance à l'Europe », In : BITSCH M.-T., LOTH W., POIDEVIN R. (dir.), Institutions européennes et identités européennes, Bruylant, Paris, 1998 , p. 146.

${ }^{12}$ Recommandation 1247, op. cit.

13 KLEBES H., « Le Conseil de l'Europe survivra-t-il à son élargissement ? », In : FLAUSS J.-F. et WACHSMANN P., Le droit des organisations internationales, Bruxelles : Editions Bruylant, 1997, p. 181.

${ }^{14}$ Vassili Tatichtchev (1686-1750), cartographe du Tsar Pierre Le Grand, définit la limite entre l'Europe et l'Asie sur les monts Oural et les plaines de la Caspienne en 1703, repoussant l'ancienne limite du Don de plusieurs milliers de km à l'est afin d'ancrer Moscou dans l'Europe dans un processus de modernisation de l'Empire russe.
} 


\section{Le spectaculaire élargissement du Conseil de l'Europe dans un contexte géopolitique nouveau}

1989 - 2003
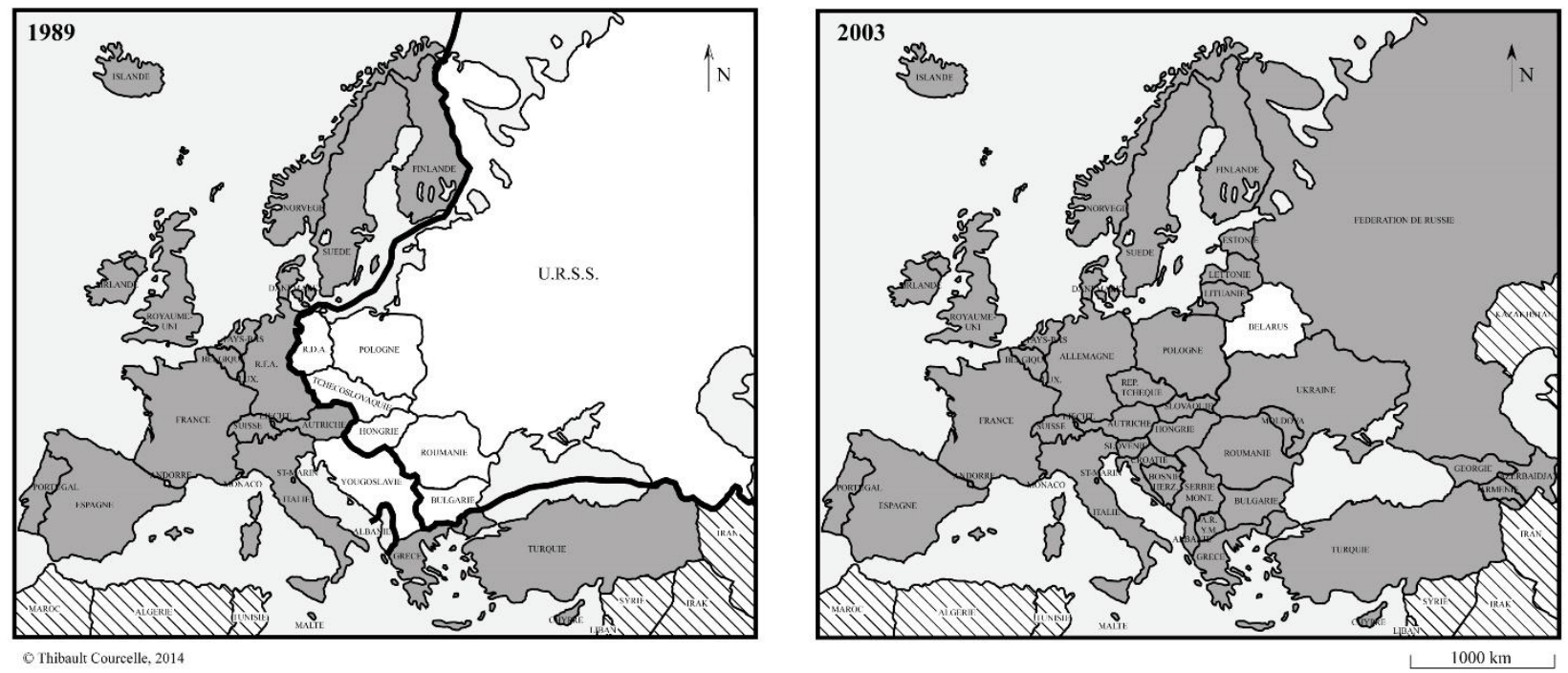

o Thibault Courcelle, 2014

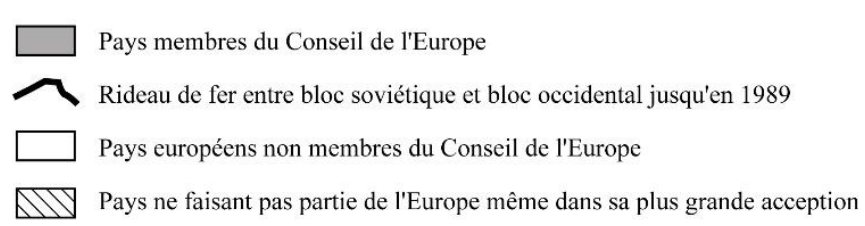

Source : Dates de ratifications sur le site du Conseil de l'Europe dans la rubrique "Etats membres" : www.coe.int

Cet élargissement rapide et spectaculaire de l'organisation pose de nombreux problèmes d'adaptation des institutions, notamment en raison de l'engagement des nouveaux membres de ratifier la Convention européenne des droits de l'homme, ce qui augmente le nombre de citoyens européens bénéficiant du droit de recours individuel contre leur propre Etat de moins de 400 millions de personnes à plus de 800 millions en à peine plus de dix ans. Cette évolution provoque en quelques années la saturation de la Cour européenne des droits de l'homme dont la situation, malgré quelques réformes, devient critique au début des années 2000. Cet élargissement permet cependant au Conseil de l'Europe de s'enorgueillir d'être devenu l'organisation de la «grande Europe » comprenant 47 Etats membres de l'Atlantique au Pacifique. D'après les critères définis par l'Assemblée parlementaire, deux Etats considérés comme pleinement européens ne sont, à ce jour, pas membres de l'organisation : la Biélorussie et le Kosovo. Leur adhésion n'est pas à l'ordre du jour. La candidature de la Biélorussie a été gelée par l'Assemblée parlementaire depuis 1997 en raison du régime autoritaire d'Alexandre Loukachenko au pouvoir depuis 1994. L'adhésion du Kosovo est pour le moment impossible en raison de la non-reconnaissance internationale de cet Etat en tant qu'Etat souverain par certains Etats européens ${ }^{15}$ depuis sa proclamation d'indépendance en 2008.

\footnotetext{
${ }^{15}$ En 2015, soit sept ans après sa création, douze Etats membres du Conseil de l'Europe sur quarante-sept (dont cinq membres de l'UE) s'opposent pour différentes raisons (crainte de mouvement sécessionniste, lien orthodoxe...) à la reconnaissance du Kosovo : Chypre, Espagne, Grèce, Roumanie, Slovaquie, Arménie, Azerbaïdjan, Moldavie, Russie, Ukraine, Géorgie et Serbie.
} 


\section{II - La politique de voisinage de l'UE, un règlement du problème des frontières pour l'organisation?}

L'UE, organisation beaucoup plus intégrée que le Conseil de l'Europe, se trouve également confrontée au problème de ses propres frontières suite au vaste élargissement à l'Est de 2004 et 2007 à douze nouveaux Etats membres, dont dix d'Europe centrale et orientale, considéré comme révolutionnaire de par son ampleur et sa valeur de symbole d'effacement de la guerre froide. Cet évènement marque la naissance d'une nouvelle UE car l'intégration de ces Etats, beaucoup moins riches que les anciens membres, provoque un profond bouleversement dans la répartition des aides de la politique de cohésion, servant à corriger les déséquilibres des nouveaux entrants dans l'UE qui présentent d'importants écarts régionaux vis-à-vis des anciens membres. Malgré 351,8 milliards d'euros alloués pour la dernière période de programmation 2014-2020 ${ }^{16}$, l'homogénéisation du développement des territoires européens par la convergence économique et sociale est difficile à réaliser depuis les élargissements à l'Est tant les disparités de développements socio-économiques sont importantes. La superficie de l'UE s'accroît de près de $25 \%$ et sa population de $20 \%$ en passant de 375 à 450 millions d'habitants, mais son PIB n'augmente que de $5 \%{ }^{17}$. L'hétérogénéité de l'UE en termes économiques et les écarts entre régions riches et régions pauvres sont donc nettement plus importants qu'auparavant, ce qui implique un processus de rattrapage nécessairement long.

Pour définir ses propres frontières, l'UE n'a pas, comme le Conseil de l'Europe, adopté un texte mais a défini une politique de partenariat spécifique pour délimiter une zone frontalière tampon entre ses Etats membres et l'extérieur. La réflexion sur la mise en place de cette politique commence dès 1998, suite à l'ouverture des négociations d'adhésion avec les Etats d'Europe centrale. Plusieurs Etats de l'UE, en particulier le R-U, la Suède et l'Allemagne, proposent des initiatives politiques vers l'Est appelées «Europe élargie» (Wider Europe). L'idée est de proposer une politique sous-régionale aux Etats d'Europe orientale sur le modèle du partenariat euroméditerranéen créé en 1995 avec le Processus de Barcelone. L'initiative est ensuite reprise par la Pologne et la Lituanie qui font du lobbying auprès du Conseil et de la Commission européenne avant même leur adhésion (dès juin 2001 pour la Pologne). Ces Etats envoient plusieurs documents informels (non papers) appuyant le développement d'une politique européenne vers l'Est "permettant d'inciter d'éventuels candidats, l'Ukraine en tête, à engager des réformes destinées à les mettre sur la voie de l'adhésion ${ }^{18}$. Un tropisme géographique et culturel pousse les nouveaux membres à encourager la poursuite des élargissements de l'UE vers l'Est même si le degré d'engagement de ces Etats envers leurs voisins est loin d'être homogène. La Pologne et les Etats Baltes soutiennent l'adhésion de l'Ukraine et de la Géorgie, la Roumanie celle de la Moldavie, d'où le concept d' «Europe élargie » qui est mis en avant. La République tchèque et la Hongrie, qui n'ont pas de frontières directes avec l'Europe orientale, s'intéressent plus tardivement à un rapprochement de l'UE vers l'Europe orientale. Les nouveaux membres ne se présentent donc pas comme un bloc uni.

Dans la communication des institutions européennes, le terme d' «Europe élargie » utilisé jusqu'en 2002 cède rapidement la place à celui, moins engageant en terme d'adhésion, de «Politique européenne de voisinage (PEV)» (the European Neighbourhood Policy) à partir de 2003. Ce glissement sémantique n'est pas anodin. L'UE marque clairement une différenciation entre

\footnotetext{
${ }^{16}$ COURCELLE T., TAULELLE F., «La citoyenneté européenne est-elle favorisée par la politique de cohésion ? », In : CATALA M., JEANNESSON (dir.), L'Europe des citoyens et la citoyenneté européenne : évolutions, limites, perspectives, PIE - Peter Lang, Bruxelles, à paraître en 2015.

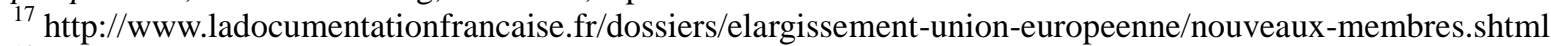

${ }^{18}$ TULMETS E., «Les «nouveaux » Etats membres et la politique européenne de voisinage », Annuaire Français de Relations Internationales, Volume X, 2009, p. 6.
} 
«son» territoire et celui des «voisins », ce qui n'était pas le cas avec l'expression d' «Europe élargie » plus englobante. La fonction du discours est donc ici de construire de nouveaux voisins, un «cercle de pays amis » pour éviter l'édification d'un nouveau « rideau de fer » entre l'UE et ses voisins ${ }^{19}$. Pour Javier Solana, haut représentant de l'UE pour la politique étrangère et la sécurité commune, ce nouveau concept de voisinage se justifie par le contexte géopolitique mondial au début du XXIè s. :

"Même à l'heure de la mondialisation, la géographie garde toute son importance. Il est dans l'intérêt de l'Europe que les pays situés à ses frontières soient bien gouvernés. Les voisins engagés dans des conflits violents, les Etats faibles où la criminalité organisée se répand, les sociétés défaillantes où une croissance démographique explosive aux frontières de l'Europe constituent pour elle autant de problèmes. Si elle accrô̂t notre sécurité, l'intégration des Etats adhérents aura également pour effet de rapprocher l'UE des zones de troubles. Notre tâche doit être de promouvoir, à l'Est de l'Union européenne et aux frontières du bassin méditerranéen, un ensemble de pays bien gouvernés avec lesquels nous pourrons avoir des relations étroites, fondées sur la coopération. ${ }^{20}$

C'est donc la dimension sécuritaire qui prime dans les préoccupations de l'UE, bien que la PEV comprenne aussi des volets de coopération économique et culturelle.

Le problème des opinions publiques au sein des anciens Etats membres, où l'enthousiasme des années 1990 a laissé place à une appréhension et à des craintes liées à l'élargissement, se pose aussi avec une certaine acuité (Ex. les représentations en France sur le plombier polonais venant concurrencer le plombier français lors du référendum sur le traité constitutionnel en 2005). Il n'y a pas eu de consultation de la population des pays déjà membres sur ces élargissements, alors que la population des nouveaux membres a été consultée en 2003. Les sondages de l'Eurobaromètre, institution au service de la Commission européenne, permettent cependant d'avoir une idée relativement précise des représentations des opinions publiques des anciens membres envers de futurs élargissements. Le sondage Eurobaromètre Flash 140 de mars 2003 portant sur l'élargissement montre une attente de la part des citoyens de l'UE quant à la nécessité de mener une réflexion sur l'avenir de l'UE. En moyenne, les trois quarts des citoyens de l'UE pensent qu'avant tout nouvel élargissement, il sera nécessaire de se mettre d'accord sur les frontières définitives de l'UE et $69 \%$ des répondants se disent «plutôt d'accord » avec le fait que l'UE devrait développer un autre type de relation qui ne va pas aussi loin que l'adhésion pour d'autres pays voisins ${ }^{21}$. Ces résultats nous indiquent que les citoyens de l'UE, avant même le grand élargissement de 2004, souhaitent une clarification préalable des limites géographiques de l'UE ainsi que le développement d'alternatives à l'adhésion. Les questions posées dans ce sondage ne sont pas anodines, puisque la Commission européenne lance alors formellement la politique européenne de voisinage (PEV) en mars 2003 par une communication de la Commission intitulée «L'Europe élargie - Voisinage », et cherche ainsi à légitimer cette politique.

Les défis externes de géopolitique pour l'UE posés par les élargissements avec le rapprochement de zones appauvries et très conflictuelles (Ukraine, Moldavie, Caucase du sud, Proche-Orient...) sont immenses. Ils impliquent également une redéfinition des relations entre l'UE et la Fédération de Russie qui partagent depuis 2004 près de 2300 kilomètres de frontière commune. Ces élargissements de l'UE, précédés pour les nouveaux Etats membres d'une intégration à l'OTAN, ont été perçus par les dirigeants et la population en Russie comme une perte de l'influence russe dans son ancien «pré carré soviétique ». La montée en puissance de la Russie dans les années 2000, grâce à l'envolée du prix du gaz et du pétrole permettant un redressement économique et militaire, s'est accompagnée d'une montée des tensions avec l'UE lors de plusieurs évènements (révolutions

\footnotetext{
${ }^{19}$ PATTEN C., PRODI R., «A côté de l’Union européenne, un cercle de pays amis », Le Monde, 19 mars 2003.

${ }^{20}$ SOLANA J., «Une Europe sûre dans un monde meilleur », Stratégie européenne de sécurité, 12 décembre 2003 , p. 7 et 8. http://www.consilium.europa.eu/uedocs/cmsUpload/031208ESSIIFR.pdf

${ }^{21}$ http://ec.europa.eu/public_opinion/flash/fl140_fr.pdf
} 
de couleur en 2003/2004 en Géorgie et en Ukraine, indépendance du Kosovo et conflit Géorgie/Russie en 2008, crise du gaz en Ukraine en 2009, puis guerre civile ukrainienne en 2014). Dans un entretien au journal Le Monde en 2005, Vaclav Havel faisait part de la nécessité pour l'UE et la Russie de délimiter définitivement leurs frontières et zones d'influence pour apaiser les tensions : "Dans l'Histoire, la Russie s'est étendue et s'est rétractée. La plupart des conflits trouvent leur origine dans des querelles de frontières et dans la conquête ou la perte de territoire. Le jour où nous conviendrons dans le calme où termine l'Union européenne et où commence la Fédération russe, la moitié de la tension entre les deux disparaîtra. ${ }^{22}$ Peut-on, dès lors, considérer la création d'une politique européenne de voisinage par l'UE comme étant une tentative de fixer définitivement ses propres frontières? Quelles sont les limites occidentales de l'influence russe qui conviennent à l'UE ?

L'UE s'affirme alors porteuse d'une politique étrangère qui repose sur deux orientations : une approche unifiée des relations de l'Union avec l'ensemble de ses voisins, couplée à une stratégie d'intégration sans perspective d'adhésion : "Reconnaissant que « l'Union ne peut s'élargir indéfiniment ${ }^{23}$, la PEV est l'espace privilégié où se trame une forme plus subtile, plus flexible, plus difficile à coordonner, de gestion des frontières externes de l'UE ${ }^{24}$. Ceci explique que la PEV n'ait pas été proposée aux Etats des Balkans issus de l'ex-Yougoslavie, ainsi qu'à l'Albanie, car l'UE et ses Etats membres considèrent qu'ils ont tous vocation à rejoindre à terme l'organisation. Pour les mêmes raisons, la Turquie a refusé de faire partie de cette PEV puisqu'elle souhaite faire pleinement partie de l'UE depuis sa demande formelle d'adhésion à la CEE en 1987. En revanche, le Maroc, qui s'était également porté candidat à la CEE en 1987 et dont la demande avait été rejetée, est pleinement intégré dans cette PEV ainsi que l'ensemble des Etats bordant la Méditerranée. Le volet oriental de la PEV comprend l'ensemble des Etats d'Europe orientale et du Caucase reconnus comme «européens » par le Conseil de l'Europe. En catégorisant qui est membre et qui est voisin, l'UE, par l'exclusion, renforce ainsi les traits spécifiques de son identité : "définir le voisin à travers la réflexion sur les limites extérieures de l'Europe participe donc indirectement à la représentation de soi [...]. Le voisin demeure une déclinaison de l'étranger n'étant "pas complètement des nôtres, sans être totalement des autres ${ }^{25}$.

Le service d'action extérieur de l'UE, créé en 2011, présente les objectifs très généreux de la $\mathrm{PEV}$, qui offre à l'UE les moyens de renforcer les relations bilatérales avec ses voisins. Il est expliqué que cette politique s'appuie sur un engagement mutuel en faveur de valeurs communes telles que la démocratie, les droits de l'homme, l'État de droit, la bonne gouvernance, les principes de l'économie de marché et le développement durable. La PEV ne se limite pas à la mise en place d'accords de coopération ou de commerce, et permet également une association politique, une intensification de l'intégration économique, une amélioration de la mobilité et un renforcement des contacts entre les peuples ${ }^{26}$. Pourtant, dans ce partenariat asymétrique, c'est l'UE qui décide de ce que sont les «valeurs communes », et c'est aussi l'UE qui juge de la qualité des efforts consentis par le voisin pour les adopter et qui conditionne ainsi le versement de fonds. Le rapport de force est donc complètement en faveur de l'UE qui dicte ses normes à ses voisins, ce qui explique que la Russie ait refusé de faire partie de la PEV pour privilégier un partenariat stratégique - d'égal à égal - avec l'UE en 2005, afin d'améliorer leurs échanges commerciaux et mettre en place une coopération approfondie sur les questions de sécurité extérieure. L'étude des représentations associées à la PEV montre également une frustration des Etats d'Europe orientale qui souhaiteraient

\footnotetext{
${ }^{22}$ Citation reprise par FOUCHER M., L'Europe et l'avenir du monde, Editions Odile Jacob, 2009, p. 73.

${ }^{23}$ PRODI R., «A Wider Europe - A proximity Policy as the Key to Stability ». Discours tenu lors du 6è ECSA World Peace Conference, Bruxelles, 5-6 décembre 2002.

${ }^{24}$ BALZACQ T., "La politique européenne de voisinage, un complexe de sécurité à géométrie variable », Cultures et Conflits, n66, été 2007, p. 33.

${ }^{25}$ PETITHOMME M., «Quelle politique de voisinage pour l’Union européenne ? Entre injonctions sécuritaires et conditionnalité démocratique, la puissance normative », Politique européenne, 2009 / 02, n²8, p. 165.

${ }^{26}$ Site du Service européen pour l'action extérieure : http://eeas.europa.eu/enp/index_fr.htm
} 
pouvoir intégrer l'UE mais se sentent marginalisés : "la presse ukrainienne exprime son regret d'être placée "à la même enseigne " que les pays d'Afrique du Nord, et de ne pas être perçu comme un pays à part entière du continent européen ${ }^{27}$.

${ }^{27}$ DE LA BROSSE R., MARON F., «Administrer et communiquer la nouvelle politique européenne de voisinage », in BEURDELEY L., DE LA BROSSE R., MARON F. (dir.), L'Union européenne et ses espaces de proximité. Entre stratégie inclusive et partenariats rénovés : quel avenir pour le nouveau voisinage de l'Union ?, Bruxelles, Bruylant, 2007, p. 68. 


\section{L'Union européenne et sa politique de voisinage en 2014}

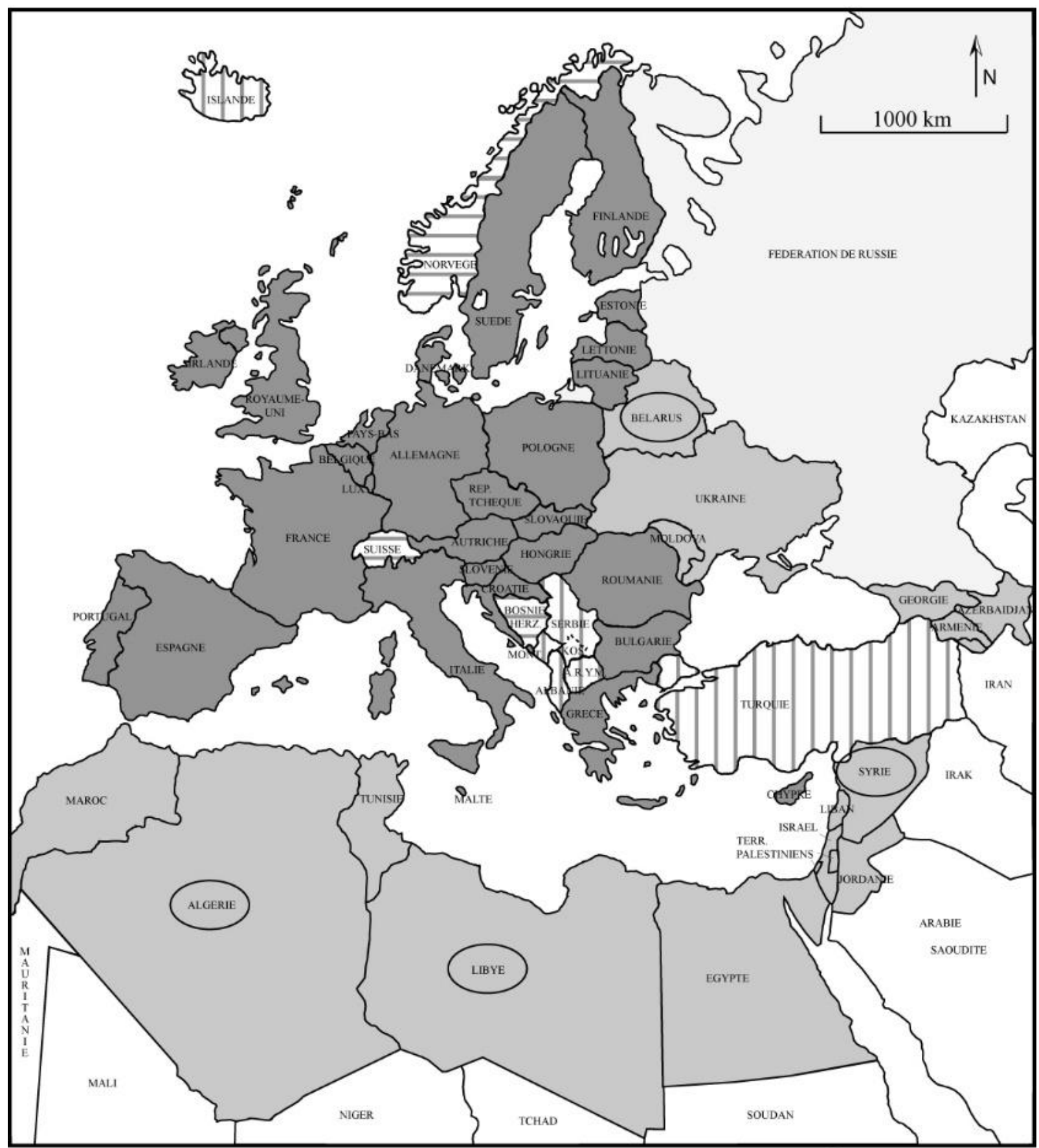

Situation des Etats vis-à-vis de l'UE :

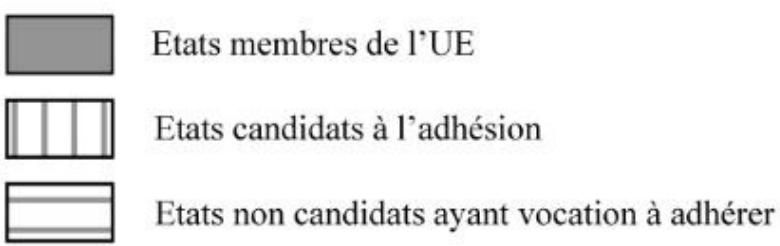

(c) Thibault Courcelle, 2014

$\square$ Partenariat stratégique UE-Russie

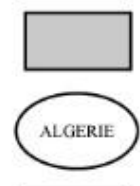

Etats couverts par la Politique européenne de voisinage

Etats n'ayant pas encore activé la PEV

Autres Etats

Au regard des enjeux géopolitique de sécurisation et de stabilisation des frontières extérieures, le budget de la PEV est assez limité puisque, pour la période budgétaire 2007/2013, son financement s'élève à près de 12 milliards d'euros, soit 2 milliards d'euros par an, à peine plus de $1 \%$ du budget total de l'UE. Ce budget est légèrement supérieur pour la période 2014/2020 avec plus de 
15 milliards d'euros, ce qui traduit une relative montée en puissance de la PEV. Néanmoins, ce faible budget rapporté à seize pays potentiellement bénéficiaires, est loin de répondre aux principaux objectifs fixés par l'UE pour la PEV. Si la Commission reconnaît quelques progrès dans certains domaines - les échanges commerciaux ont progressé de $63 \%$ pour les exportations et de $91 \%$ pour les importations avec les pays du voisinage entre 2005 et $2010^{28}$ et de nombreux accords sur l'énergie ont été signés garantissant une meilleure sécurité énergétique pour l'Europe - elle constate également que ces progrès touchent inégalement les pays de la PEV et que certains sont en régression, notamment sur la question des droits fondamentaux. Suite aux révolutions arabes de 2011, l'UE adopte une «nouvelle PEV » qui prévoit des aides accrues en échange de progrès démocratiques ou de réformes économiques et qui met particulièrement l'accent sur un partenariat renforcé avec la société civile «élargie » (associations, étudiants, secteur éducatif, entreprises etc.), dans la réalisation de projets communs.

L'actualité de ces derniers mois en Ukraine démontre que la PEV est insuffisante face aux enjeux géopolitiques du voisinage de l'UE. Le début des troubles dans ce pays est directement lié au choix du gouvernement ukrainien de Viktor Ianoukovitch, le 21 novembre 2013, de refuser, sous la pression de la Russie, le renouvellement d'un accord d'association avec l'UE dans le cadre de la PEV pour privilégier un accord de partenariat économique avec la Russie et les Etats de la CEI. Ce refus a entraîné d'importantes manifestations pro-européennes à Kiev rassemblant des centaines de milliers de personnes sous la bannière étoilée de l'Europe et l'occupation de la place de l'indépendance - place Maïdan - et de la mairie, avec comme mot d'ordre la démission du président Ianoukovitch. Les violences du 18 au 21 février qui ont fait plus de 80 morts ont marqué un tournant dans ce conflit avec pour conséquence la destitution du président et avec comme contrecoup, l'annexion de la Crimée par la Russie et le déclenchement d'une guerre civile dans la région du Donbass à l'Est. Depuis le mois d'avril 2014, ce conflit a fait plus de 3600 morts et plus de 8000 blessés, ainsi qu'entre 659000 et 921000 déplacés et plusieurs centaines de milliers de réfugiés de l'Ukraine vers la Russie d'après le gouvernement ukrainien ${ }^{29}$.

\section{Conclusion :}

Evoquer la question des frontières de l'Europe c'est, pour les institutions européennes, un moyen de s'interroger sur les limites finales souhaitables de l'Europe confédérale (la "grande Europe » du Conseil de l'Europe) et de l'Europe instituée (celle plus restreinte de l'UE). Si la question des critères d'européanité et donc des limites a été fixée par le Conseil de l'Europe en 1994 et ne semble pas être remise en cause, plusieurs représentations géopolitiques s'opposent et divisent les Etats, les partis politiques et les opinions publiques quant à la question des frontières de l'UE et de ses futurs élargissements. La question posée est donc bien celle de la finalité du projet européen et du degré d'intégration entre les membres de l'UE. Si cette question a une forte dimension géographique, elle relève d'abord de débats et de décisions politiques, et dépend beaucoup de l'évolution du contexte géopolitique international : "Selon le critère retenu pour définir l'identité politique et le projet géopolitique européens, le scénario qui en découle est différent ${ }^{30}$. Pour 1'UE, la politique européenne de voisinage lancée en 2004 est une tentative d'apporter une réponse à la question de ses frontières futures.

A moyen terme, l'UE s'élargira probablement à l'ensemble des Balkans et comptera environ 35 membres. Les quatre Etats membres de l'Association européenne de libre-échange (AELE) Norvège, Islande, Suisse et Liechtenstein - ont aussi la possibilité de rejoindre l'UE, ce qui, dans ce cas, porterait à une quarantaine le nombre de ses membres. Il n'est plus improbable que certains

\footnotetext{
${ }^{28}$ EU Neighbourhood info center : http://www.enpi-info.eu/mainmed.php?id=21854\&id_type=1

${ }^{29}$ Agence des Nations Unies pour les réfugiés : http://www.unhcr.fr/54c256b9c.html

${ }^{30}$ FOUCHER M., «L'Europe, une géopolitique du mouvement », in FOUCHER M. (dir.), L'Europe entre géopolitiques et géographies, SEDES/CNED, Paris, 2010, p. 55.
} 
Etats comme le Royaume-Uni, sous la pression de partis eurosceptiques, quittent l'UE. Le premier ministre britannique David Cameron a promis un referendum sur le Brexit (British exit) dès 2016 en cas de victoire des conservateurs lors de l'élection du 7 mai 2015. L'intégration de la Turquie, dont les négociations d'adhésion ont commencé il y a dix ans, semble très hypothétique, pas seulement en raison des nombreux points d'achoppement dans les négociations (Chypre, relations avec la Grèce, question kurde...), mais surtout à cause des opinions publiques européennes et des gouvernements de plus en plus réticents, ainsi qu'une part importante de la société turque opposée à cette adhésion. La Turquie sera probablement, à terme, intégrée dans la politique européenne de voisinage.

L'UE s'oriente donc vers un scenario de stabilisation de ses frontières, où, hormis l'intégration progressive des Balkans, elles n'évolueraient plus, ce qui implique un renforcement à l'avenir du partenariat à l'Est et au Sud dans le cadre de la PEV, avec des coopérations transfrontalières sur le modèle des coopérations internes à l'UE. La géographie des frontières ultimes de l'UE est dans sa politique et ses décisions. Il est important de fixer des bornes à cette institution géopolitique pour mieux redéfinir ses finalités et redonner du sens au projet européen.

\section{Bibliographie :}

BALZACQ T., «La politique européenne de voisinage, un complexe de sécurité à géométrie variable », Cultures et Conflits, $\mathrm{n}^{\circ} 66$, été 2007, p. 31 à 59.

BEURDELEY L., DE LA BROSSE R., MARON F. (dir.), L'Union européenne et ses espaces de proximité. Entre stratégie inclusive et partenariats rénovés : quel avenir pour le nouveau voisinage de l'Union?, Bruxelles, Bruylant, 2007, 372 p.

BITSCH M.-T., LOTH W., POIDEVIN R. (dir.), Institutions européennes et identités européennes, Bruylant, Paris, 1998, 523 p.

COURCELLE T., Le Conseil de l'Europe : enjeux et représentations, Thèse de doctorat, Institut Français de Géopolitique, Université Paris 8, 2008, 576 p.

FLAUSS J.-F. et WACHSMANN P., Le droit des organisations internationales, Bruylant, Bruxelles, 1997, 362 p.

FOUCHER M., L'Europe et l'avenir du monde, Editions Odile Jacob, 2009, 141 p.

FOUCHER M. (dir.), L'Europe entre géopolitiques et géographies, SEDES/CNED, Paris, 2010, $249 \mathrm{p}$.

HUBER D., Une décennie pour l'Histoire: le Conseil de l'Europe 1989 - 1999, Editions du Conseil de l'Europe, Strasbourg, 314 p.

MORIN E., Penser l'Europe, Gallimard, Paris, 1987, 221 p.

PETITHOMME M., «Quelle politique de voisinage pour 1'Union européenne ? Entre injonctions sécuritaires et conditionnalité démocratique, la puissance normative », Politique européenne, 2009 / 02, n²8, p. 163 à 172.

TULMETS E., «Les «nouveaux » Etats membres et la politique européenne de voisinage », Annuaire Français de Relations Internationales, Volume X, 2009, 15 p. 